\title{
Systematic review of clinical practice guidelines recommendations about primary cardiovascular disease prevention for older adults
}

Jesse Jansen ${ }^{1,2^{*}}$, Shannon McKinn ${ }^{1,2}$, Carissa Bonner ${ }^{1,2}$, Les Irwig $^{1}$, Jenny Doust ${ }^{1,3}$, Paul Glasziou ${ }^{1,3}$, Brooke Nickel ${ }^{1,2}$, Barbara van Munster ${ }^{4,5}$ and Kirsten McCaffery ${ }^{1,2}$

\begin{abstract}
Background: Clinical care for older adults is complex and represents a growing problem. They are a diverse patient group with varying needs, frequent presence of multiple comorbidities, and are more susceptible to treatment harms. Thus Clinical Practice Guidelines (CPGs) need to carefully consider older adults in order to guide clinicians. We reviewed CPG recommendations for primary cardiovascular disease (CVD) prevention and examined the extent to which CPGs address issues important for older people identified in the literature.

Methods: We searched: 1) two systematic reviews on CPGs for CVD prevention and 2) the National CPG Clearinghouse, G-I-N International CPG Library and Trip databases for CPGs for CVD prevention, hypertension and cholesterol. We conducted our search between April and December 2013. We excluded CPGs for diabetes, chronic kidney disease, HIV, lifestyle, general screening/prevention, and pregnant or pediatric populations. Three authors independently screened citations for inclusion and extracted data. The primary outcomes were presence and extent of recommendations for older people including discussion of: (1) available evidence, (2) barriers to implementation of the $C P G$, and (3) tailoring management for this group.

Results: We found 47 eligible CPGs. There was no mention of older people in 4 (9\%) of the CPGs. Benefits were discussed more frequently than harms. Twenty-three CPGs (49\%) discussed evidence about potential benefits and 18 (38 \%) discussed potential harms of CVD prevention in older people. Most CPGs addressed one or more barriers to implementation, often as a short statement. Although 27 CPGs (58 \%) mentioned tailoring management to the older patient context (e.g. comorbidities), concrete guidance was rare.

Conclusion: Although most CVD prevention CPGs mention the older population to some extent, the information provided is vague and very limited. Older adults represent a growing proportion of the population. Guideline developers must ensure they consider older patients' needs and provide appropriate advice to clinicians in order to support high quality care for this group. CPGs should at a minimum address the available evidence about CVD prevention for older people, and acknowledge the importance of patient involvement.
\end{abstract}

Keywords: Aging, Cardiovascular diseases, Guidelines, Shared decision making

\footnotetext{
* Correspondence: jesse.jansen@sydney.edu.au

'Screening \& Test Evaluation Program (STEP), Sydney School of Public Health,

The University of Sydney, Edward Ford Building A27, Sydney NSW 2006,

Australia

${ }^{2}$ Centre for Medical Psychology and Evidence-based Decision-making

(CeMPED), The University of Sydney, Edward Ford Building A27, Sydney NSW

2006, Australia

Full list of author information is available at the end of the article
} link to the Creative Commons license, and indicate if changes were made. The Creative Commons Public Domain Dedication waiver (http://creativecommons.org/publicdomain/zero/1.0/) applies to the data made available in this article, unless otherwise stated. 


\section{Background}

\section{Available evidence for older people}

It is a major challenge for clinicians to provide appropriate and patient centered care for older people with comorbidities. Clinical practice guidelines (CPGs) aim to support clinician decision making, however, CPGs may not always be straight forward to implement for older people for several reasons [1]. Older people have generally been excluded from clinical trials, and when included they are generally more fit and healthy than the older people in the community [2,3]. Furthermore, clinical trials often do not address outcomes that may have high priority for older adults (e.g. quality of life and independent living) [4]. Moreover, most CPGs focus on a single disease, however the prevalence of comorbidities increases with age and studies in Australia [5] and Scotland [6] estimate that around 70 percent of people over 75 have two or more chronic conditions. Applying CPGs for each condition leads to polypharmacy, increases treatment burden, and risk of adverse events [1].

\section{Barriers to implementation of CPGs}

There are other barriers to applying CPGs to older people. Treatment in older populations is generally more complex due to comorbidities and associated polypharmacy. Data from the US and Australia suggest that over $90 \%$ of older persons (75 years and above) take one or more prescription medication, with approximately $40 \%$ of older persons using five or more prescription medications at one time $[7,8]$. Older people who take multiple medications are at increased risk of experiencing adverse drug reactions [9] and the presence of comorbidities and polypharmacy also means that it can be difficult to predict the effect of a treatment, and compare the overall benefits and harms [10]. Moreover, older people are heterogeneous in terms of general health status, frailty and cognitive function and even older people with the same diagnosis may therefore respond different to treatment [4]. Older people will also vary in terms of prognosis, and when applying CPG recommendations to older people it is important to determine if the person is likely to benefit from the medication within their remaining life (time needed to treat to benefit) $[11,12]$. Moreover, they will vary in their treatmentand health outcome preferences (e.g. length of life versus quality of life, physical and cognitive functioning, risk reduction, tolerance of side effects) [13-15].

\section{Tailoring treatment to older patient context, preferences and goals}

Decision making about initiating treatment in older people therefore requires careful tailoring to the individual patient's circumstances and it is critical to involve the older patient and take their preferences into account in the shared decision. Older patients' preferences and treatment goals are are more variable than younger people, and likely to change depending on factors such as health and mood $[15,16]$. This highlights the importance of eliciting patient preferences when deciding on treatment in this group. Most people, including older people [17], prefer to be involved in the decision making process. The majority of older people want to discuss options and receive information even though they may not wish to be involved in making the final decision [18] and value the input of their family member/carer who are often present in the consultation [19]. However, CPGs are often not set up in a way that optimally supports patient involvement and shared decision making [20], this is a general limitation although it is probably particularly relevant for older people.

\section{Need for guidance primary CVD prevention in older people}

In this paper we focus on CPGs for primary cardiovascular disease (CVD) prevention in older people. Worldwide, the population is aging and because CVD risk increases with age, this creates a major public health burden. Moreover, medication use for CVD prevention is common in older people, for example one-third of people aged 75-84 in Sweden [21] are treated with statins and in the US over $80 \%$ of adults with hypertension aged 60 or older receive anti-hypertensive medication [22]. This means that there is a clear imperative for CPG for primary CVD prevention to address older people. Most international cardiovascular disease (CVD) primary prevention CPGs encourage the use of 5- or 10 year absolute (or overall, global or combined) CVD risk scores (AR) to target preventive treatment in asymptomatic patients who are at high risk [23]. However, CVD risk prediction models are not well validated in older people, for example the widely used Framingham risk equation is based on a patient cohort with an upper age of 74 [24]. Because AR increases with age, and people at higher risk benefit more in terms of risk reduction, it is reasonable to argue that older people (and especially otherwise healthy older people) have the potential to benefit at least as much from primary CVD prevention as younger people $[25,26]$ and otherwise healthy older people should not be denied potentially effective preventative medication based on their age alone. However, although there is some evidence of the benefits of blood pressure $[27,28]$ and cholesterol lowering medication in older people $[29,30]$, most of these studies did not take into account comorbidities [31]. Also, the harms of medication (e.g. myopathy with cholesterol medication and risk of falls with blood pressure medication) are more likely to occur in older people [31-34] and older people are likely to vary widely in the relative importance they place on benefits and harms of CVD medication [15]. Moreover, not every older patient will be able to achieve benefits from long-term preventative CVD medication during their remaining life span [12]. 
In summary, when making decisions about primary CVD prevention for older people, clinicians have the challenging task of weighing AR with life expectancy, comorbidities, the benefits and harms of medication, and preferences of patients and family members/carers. Not surprisingly, clinicians have reported the need for clearer guidance in this area [35]. The purpose of this paper is to systematically review international CPGs for primary CVD prevention to examine the extent to which they address older adults and take into account factors important for patient-centered care of older people that we identified from the literature $[1,4,13,36,37]$ as described above: available evidence for older people, barriers to implementation of the CPG for older people, and tailoring treatment to older people context and preferences. Studies looking at Australian and Canadian CPGs suggest that only a few provide recommendations tailored to the special needs of older people [36, 37]. As far as we are aware, no studies have examined CPG recommendations for primary CVD prevention in older people.

\section{Methods}

Data sources

First, we searched two systematic reviews on CPGs for CVD prevention $[23,38]$ for CPGs discussing a combined, overall, global, total or absolute risk approach (from here on referred to as AR approach) and looked for updated version of these CPGs. Second, we performed our own systematic review to search for CPGs published after May 2009, the time-frame used in the previous studies, by searching the following three CPG-specific databases: the National CPG Clearinghouse (http://www.guideline.gov), G-I-N International CPG Library (http://www.g-i-n.net) and Trip database (https://www.tripdatabase.com). These databases are publicly available on the Internet. We conducted our search between April and December 2013.

\section{Inclusion/exclusion criteria}

We included published CPGs on the assessment and/or management of CVD risk or two important risk factors for CVD: hypertension and high cholesterol. We only considered CPGs that mentioned an AR approach and specifically aimed to prevent a first CVD event (primary prevention). We excluded CPGs for diabetes, chronic kidney disease, HIV, lifestyle, general screening/prevention, pregnant or pediatric populations. We restricted the search to English language CPGs. Where more than one version of the same CPG was found we included only the most recent version.

\section{Search strategies}

An information specialist was consulted to design the search strategy. The Medline search strategy consisted of two categories of subject headings (MESH) and title words (intersected by the Boolean term "AND") covering (1) CVD, hypertension and dyslipidemia: (cardiovascular disease, heart disease, vascular disease, cardiac, coronary, ischemic, cardiovascular, cerebrovascular disorders, stroke, peripheral vascular diseases, renovascular or stroke, dyslipidemia, cholesterol, lipid, hypertension, blood pressure); and (2) clinical practice guidelines (practice guidelines, guidelines, clinical or practice and guidelines). We limited our search to English language articles. The Medline search syntax served as a basis for all search strategies. The full search strategy is available on request from the authors. We were also provided with CPGs through informal communication.

Two reviewers (JJ and SM) independently assessed all 1) titles and 2) abstracts/full text CPGs for potential eligibility, with discrepancies resolved by consensus if needed after discussion with all authors (see Fig. 1). For CPGs extracted from the two existing reviews, we combined step 1 and 2 .

\section{Outcomes}

When no specific age cut-off for older adults was indicated in the CPG, we looked for words that signified older age (e.g., senior, frail, elderly). The primary outcome was to identify the extent to which recommendations for CVD assessment/management in older adults (with or without comorbidities ) were given, more specifically any mention of: (1) available evidence for older people, (2) barriers to implementation of the CPG for older people, and (3) tailoring treatment to older people context and preferences. These criteria were selected from literature on the applicability of CPGs and patient centered care for older people [1, 4, 13, 36, 37]. See Table 1 for a more detailed description of these criteria.We only included statements that explicitly referred to older patients, which means that general statements for example about the importance of patient involvement are not reported. Data were collected, summarized, and tabulated in an Excel spread sheet.

\section{Data collection and analysis}

An initial data extraction plan was formulated (JJ), then discussed and revised with additional categories (JJ, CB, $\mathrm{KM}, \mathrm{SM}$ ), before all authors agreed on the approach. Three authors (JJ, SM, BN) independently extracted data from included CPGs and all CPGs were double coded to ensure accuracy and consistency. Any discrepancies were resolved by consensus to establish a single dataset. Data was collected on year of publication/update, principal disease, organization responsible for CPG development, country/region, whether it was an assessment CPG, a management CPG, or both. In line with the outcome measures, information was also extracted on any statements/recommendations that specifically addressed older people. 


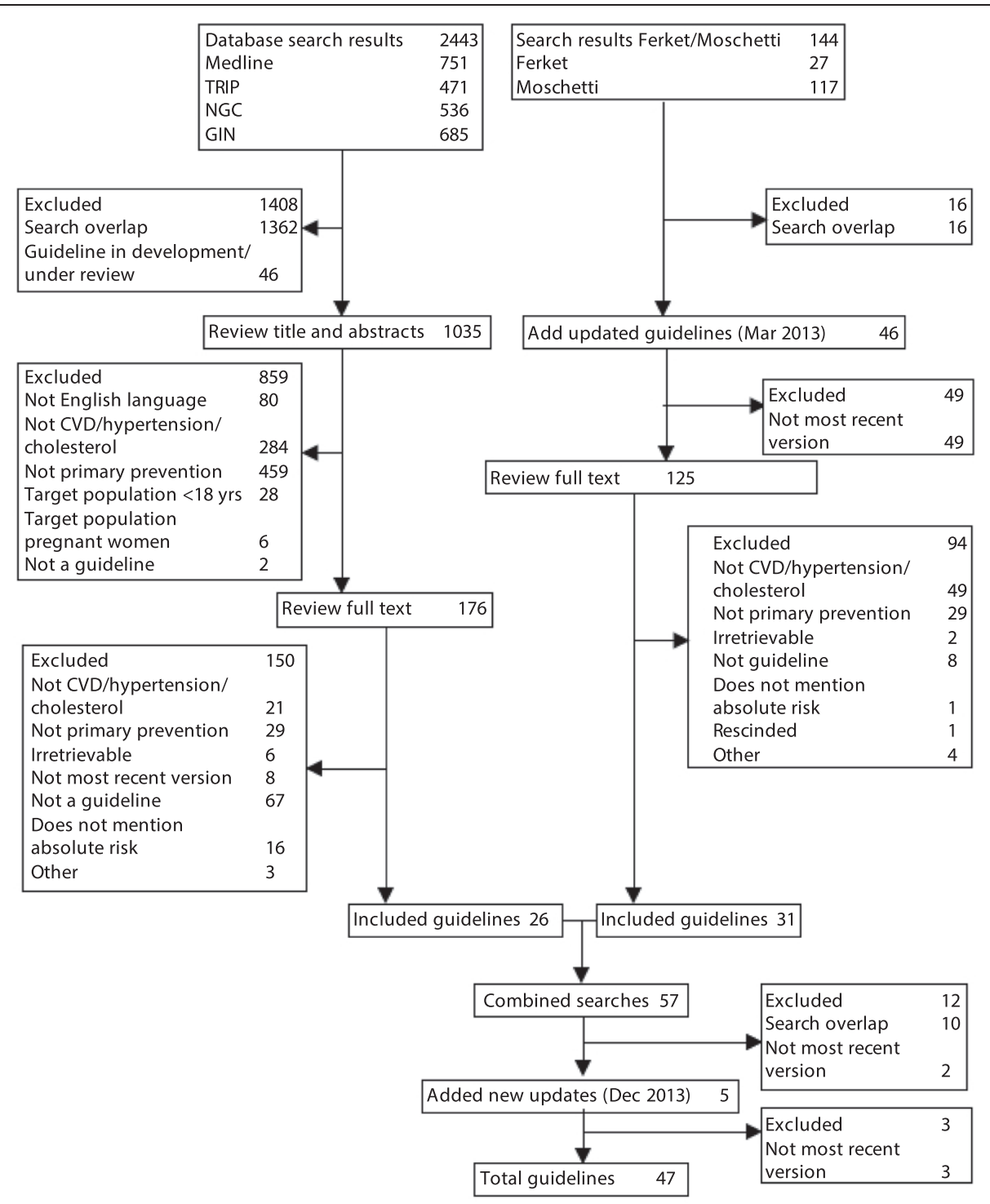

Fig. 1 Summary of CPG search and review process

\section{Results}

Of 1035 screened abstracts and 301 full text CPGs, 47 CPGs were included: 23 on assessment/management of CVD risk in general, 13 on cholesterol, and 11 on hypertension. Additional file 1: Appendix A summarizes the selected CPGs, including risk model and condition: CVD, hypertension or cholesterol. The denominator used is all CPGs $(n=47)$ unless statements specifically relate to hypertension or cholesterol (medication); in those instances the denominators are $34(23 \mathrm{CVD}+11$ hypertension CPGs) and 36 (23 CVD + 13 cholesterol CPGs), respectively.

\section{Inclusion of information related to older people}

Almost all CPGs (92\%, 43/47) specified an age range for the target population and/or provided specific recommendations for older people. The statements provided ranged from general and brief to extensive and more specific recommendations providing references to relevant evidence, although the latter was rare (see Additional file 2: Appendix B). Thirty-eight percent of the CPGs (18/47) addressed older people with comorbidities, mostly just briefly mentioning that CVD risk management in older people should take into account comorbidities without specifying specific co-occurring conditions. In addition, $21 \%(10 / 47)$ of the CPGs referred to frail older people, mostly recommending more caution with managing CVD risk in this group. Not in Table/Figure.

"People aged 75 or older should be considered at increased risk of CVD (...). Assessment and treatment should be guided by the benefits and risks of 
Table 1 Overview of criteria for CPG recommendations for older people*

\begin{tabular}{|c|c|c|}
\hline \multicolumn{3}{|l|}{ Inclusion of information related to: } \\
\hline $\begin{array}{l}\text { 1. Available evidence primary CVD prevention } \\
\text { for older people }\end{array}$ & $\begin{array}{l}\text { 2. Barriers to implementation of the CPG } \\
\text { for older people }\end{array}$ & $\begin{array}{l}\text { 3. Tailoring treatment to older people context and } \\
\text { preferences }\end{array}$ \\
\hline \multirow{8}{*}{$\begin{array}{l}\text { a. Evidence potential benefits/harms } \\
\text { b. Knowledge gaps }\end{array}$} & \multirow{2}{*}{$\begin{array}{l}\text { a. Risk assessment complexity (e.g. measurement } \\
\text { issues) }\end{array}$} & \multirow{2}{*}{$\begin{array}{l}\text { a. Patient preferences/values } \\
\text { b. Family preferences/values }\end{array}$} \\
\hline & & \\
\hline & $\begin{array}{l}\text { b. Risk management complexity (e.g. feasibility } \\
\text { treatment targets) }\end{array}$ & \multirow{2}{*}{$\begin{array}{l}\text { c. Patient context (e.g. quality of life, life } \\
\text { expectancy, comorbidities) } \\
\text { d. Weighing benefits/harms }\end{array}$} \\
\hline & $\begin{array}{l}\text { c. Time needed to treat to benefit in context of } \\
\text { life expectancy }\end{array}$ & \\
\hline & d. Meaningfulness outcomes for older people & \multirow[t]{4}{*}{ e. Therapy prioritization } \\
\hline & e. Treatment adherence issues & \\
\hline & f. Cognitive status & \\
\hline & g. Social support/caregiver burden & \\
\hline
\end{tabular}

${ }^{\circ}$ Criteria were selected from literature on the applicability of CPGs and patient centered care for older people $[1,4,13,34,35]$

treatment, informed preference and comorbidities that may make treatment inappropriate [39]".

"Initial doses and subsequent dose titration should be more gradual because of a greater chance of undesirable effects, especially in very old and frail subjects [40]".

\section{Available evidence for older people}

In total, $55 \%$ (26/47) of the CPGs discussed the available evidence for primary CVD prevention in older people (see Fig. 2 for more detail). There was a disparity between discussion of potential benefits and harms, with benefits discussed more frequently ( $49 \%$ of the CPGs, 23/47), than harms (38\% of the CPGs, 18/47). Knowledge gaps were discussed in $36 \%$ of the CPGs (17/47). Additional file 2: Appendix $B$ describes examples of brief and more extensive guideline recommendations for older people according to the three criteria for CPG recommendations in older people and Table 2 lists potential benefits and harms of different CVD risk management strategies (primary prevention) as mentioned in the CPGs.

\section{Potential benefits}

Nineteen percent (9/47) of the CPGs discussed overall benefits of CVD assessment and management in older people, mostly referring to a similar relative benefit but greater absolute benefit for older people due to higher pre-treatment risk. Benefits of medication (morbidity/ mortality benefit, positive effects on cognition) were discussed in $32 \%(11 / 34)$ of the CVD/hypertension CPGs and $33 \%$ percent $(12 / 36)$ of the CVD/cholesterol CPGs. Fifteen percent of all CPGs (7/47) discussed the benefits of lifestyle management on CVD related morbidity and mortality.

\section{Potential harms}

Overall harms of CVD risk assessment and management in older people were discussed in $17 \%(8 / 47)$ of the CPGs, mainly addressing that absolute risk assessment may underestimate risk in older people, and the use of resources for older people who are less likely to benefit due to short lifespan. Nineteen percent (7/36) of CVD/ cholesterol CPGs mentioned potential harms of cholesterol lowering medication (e.g. muscle toxicity, increased risk of cancer) whereas only $15 \%(5 / 34)$ of CVD/hypertension CPGs mentioned potential harms of blood pressure medication, mostly referring to risk of hypotension. None of the CPGs mentioned potential harms of lifestyle change in older people.

\section{Benefit harm trade-off}

Generally, the trade-off between benefits and harms of medication is more complicated in older people. Twentyfive percent (13/47) of the CPGs explicitly referred to making this trade-off, with statements varying from very general statements to more extensive discussions in a few cases (see for example New Zealand Primary Care Handbook 2012 in Additional file 2: Appendix B).

\section{Knowledge gaps}

Knowledge gaps related to CVD risk assessment and management in older people were discussed in $15 \%(7 / 47)$ of the CPGs, describing that most CVD risk models and interventions have not been thoroughly tested in older people, and the lack of generalizability of randomized clinical trials to older people in the community. Seventeen percent of the CVD/cholesterol CPGs (6/36), and $12 \%$ of the CVD/hypertension CPGs (4/34) addressed limited available evidence of treatment with preventative CVD medication for older people, especially the very old/more frail. None of 


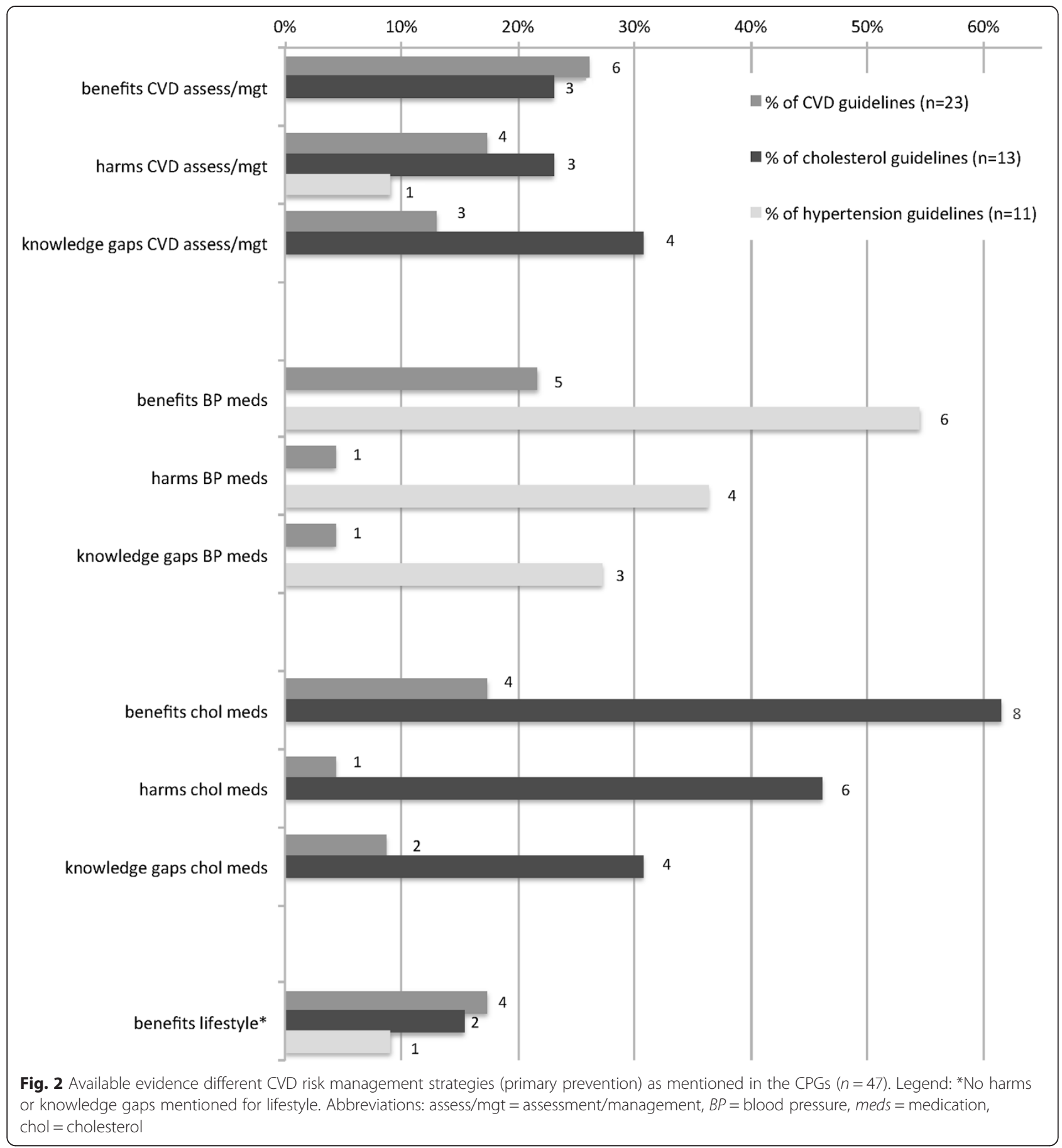

the CPGs addressed knowledge gaps around lifestyle interventions for older people.

\section{Aspirin in CVD CPGs}

Despite the lack of evidence for the use of aspirin in primary prevention of CVD [41], $9 \%(4 / 47)$ of the CPGs mentioned the potential benefits in terms of CVD morbidity/mortality and specifically reduction of myocardial infarctions. Most of these CPGs (6 \%, 3/47) also addressed the increased risk of gastrointestinal bleeds and/or hemorrhagic strokes and suggested that older people need higher baseline risk for benefits to outweigh harms (not in Figure/Table).

\section{Barriers to implementation of the CPG for older people}

The majority of CPGs addressed one or more barriers to application of the guideline in older people (83\%, 39/ 47). Details of the specific barriers or complexities that 
Table 2 Potential benefits, harms and knowledge gaps of different CVD risk management strategies (primary prevention) for older people as mentioned in the CPGs $(n=47)$ (Continued)

\begin{tabular}{|c|c|c|c|}
\hline \multirow[t]{3}{*}{ Lifestyle } & $\begin{array}{l}\text { Benefit of healthy diet, physical activity, } \\
\text { smoking, moderate alcohol intake }\end{array}$ & \multirow[t]{3}{*}{ Not discussed } & \multirow[t]{3}{*}{ Not discussed } \\
\hline & $\begin{array}{l}\text { Benefits of physical activity in older people } \\
\text { include mortality benefit, improved quality } \\
\text { of life and CVD risk reduction. }\end{array}$ & & \\
\hline & $\begin{array}{l}\text { Weight loss and reduction of salt intake } \\
\text { lowers blood pressure }\end{array}$ & & \\
\hline Aspirin & $\begin{array}{l}\text { Reduced risk of CVD events/myocardial } \\
\text { infarctions but older people need to have } \\
\text { higher baseline risk for benefits to } \\
\text { outweigh harms }\end{array}$ & $\begin{array}{l}\text { Risk of adverse effects increases with age } \\
\text { in particular gastrointestinal bleeding and } \\
\text { hemorrhagic strokes }\end{array}$ & Not discussed \\
\hline
\end{tabular}

${ }^{*} \mathrm{CVD}$ : cardiovascular disease; ${ }^{\dagger} \mathrm{RCT}$ : randomized controlled trial; ${ }^{\ddagger} \mathrm{BP}$ : blood pressure; ${ }^{5} \mathrm{SBP}$ : systolic blood pressure

were mentioned are discussed below and Additional file 3: Appendix C provides illustrative quotes from the CPGs.

\section{Risk assessment complexity}

\section{Absolute CVD risk (AR) approach}

Forty-nine percent (23/47) of the CPGs addressed the complexities CVD risk assessment in older people (Fig. 3). For AR assessment, some CPGs (6 \%, 3/47) recommended a similar approach in younger versus older people, whereas other CPGs $(11 \%, 5 / 47)$ advised to extrapolate the risk model for older people outside the target age range, either by using the upper age cut-off of the model or by providing adjusted risk scores for older people. Eleven percent of the CPGs (5/47) stated that older people above a certain age (usually $>80$ years) or older people with additional risk factors (e.g. hypertension, large pulse pressure, smokers, diabetes) can be assumed to be at high risk without a formal assessment.

\section{Blood pressure measurement}

Thirty-five percent of the CVD/hypertension CPGs (12/ 34) addressed blood pressure measurement in older people, including recommendations to measure blood pressure both lying/sitting and standing to document postural hypotension, and information about the higher predictive value of systolic blood pressure (SBP) versus diastolic blood pressure (DBP) in determining CVD risk, especially in older people.

\section{Additional tests}

Twenty-three percent (11/47) of the CPGs mentioned the use of additional tests in the assessment and management of CVD risk in older people, including anklebrachial index (ABI), ultrasound, wave velocity and echocardiography to detect possible arterial disease, cognitive tests and MRI to detect silent brain infarcts and

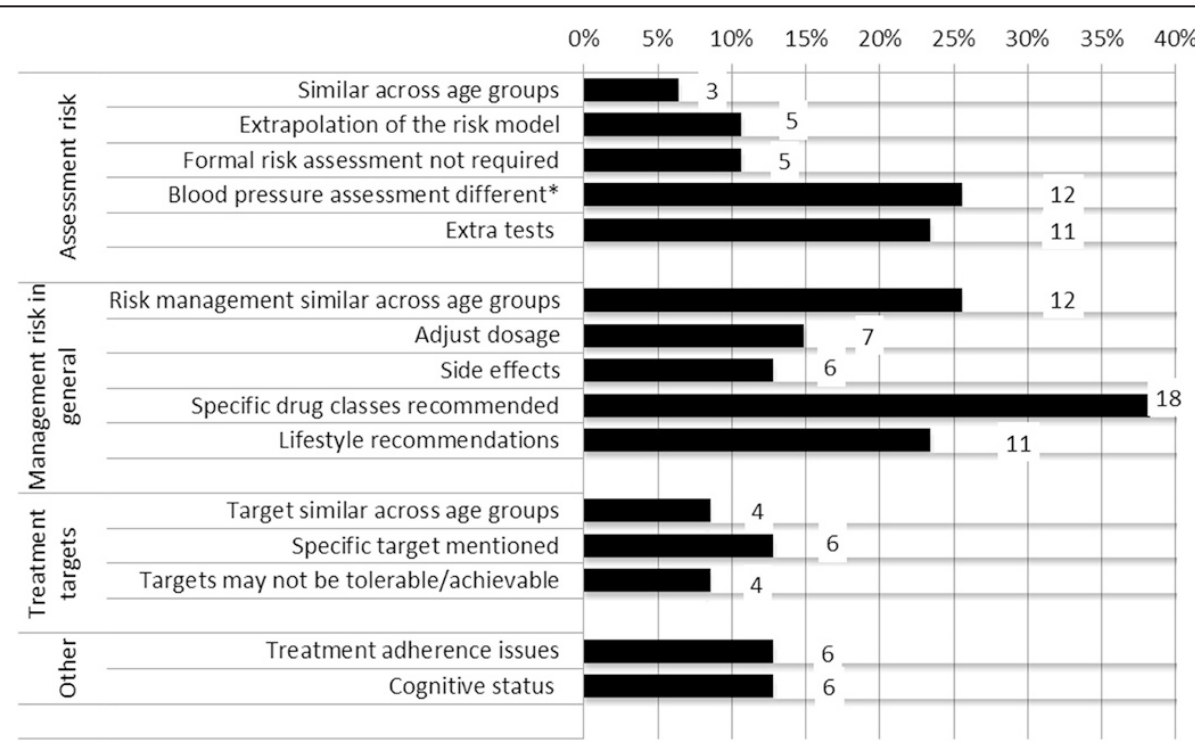

Fig. 3 Barriers to implementation of the guideline for older people as mentioned in CPGs. Legend: Percentage of total number of guidelines $n=47 ;{ }^{*}$ Calculated out of 34 (23 CVD +11 hypertension) CPGs 
creatine kinase tests before initiating treatment with cholesterol medication. This is despite the lack of good evidence for the use of tests above the Framingham risk equation.

\section{Risk management complexity Management in general}

Sixty-six percent (31/47) of the CPGs addressed management of CVD risk in older people. Twenty-six percent (12/46) stated that primary CVD prevention is generally similar for older versus younger people. However, age-specific recommendations were also provided, often by the same CPGs. Most commonly, CPGs provided a list of suitable and unsuitable pharmacotherapy (38 \%, 18/47) and lifestyle strategies $(23 \%, 11 / 47)$ for older people. Thirteen percent (6/47) addressed side effects that are more likely in older people, most notably increased risk of muscle toxicity with cholesterol lowering medication. Common recommendations for older people were to closely monitor side effects and adjust medication dosage $(15 \%, 7 / 47)$ if need be.

\section{Treatment targets}

Treatment targets for older people were addressed in $19 \%$ (9/47) of the CPGs. Some CPGs recommended similar targets for younger and older people ( $9 \%, 4 /$ 47) whereas other CPGs commented that targets may be difficult to achieve $(9 \%, 4 / 47)$ or recommended specific, less stringent targets for older people (13\%, 6/47). For example, one CPG recommended a target blood pressure below 150/90 $\mathrm{mmHg}$ in people aged 80 years and over versus $140 / 90 \mathrm{mmHg}$ in younger people [42]. None of the cholesterol CPGs discussed treatment targets for older people.

\section{Cognition and treatment adherence}

Cognition in older people was addressed in in $13 \%$ (6/ 47) of CPGs, describing the relation between hypertension/high cholesterol and cognitive impairment (see applicability of evidence) or the relation between cognitive function and adherence. Thirteen percent of the CPGs (6/47) mentioned that treatment adherence is generally lower in older people, especially in those with comorbidities and cognitive/functional impairments. One CPG recommended to improve adherence by simplifying the medication regimen. None of the guidelines discussed social support or caregiver burden.

Tailoring treatment to older people context and preferences Patient context and preferences

More than half of the CPGs (58 \%, 27/47) addressed older patient context to some extent. Most commonly (32\%, 15/ 47) CPGs recommended that the decision to assess and/or manage CVD risk in older people should be based on clinical judgment taking into account comorbidities/polypharmacy $(38 \%, 18 / 47)$, patient preferences (19 \%, 9/47), life expectancy and/or time needed to treat to benefit (19\%, 9/ $47)$, and quality of life (11 \%, 5/47). These factors were often presented in list form, instructing clinicians to apply the CPGs with consideration of individual patient context but offering no specific guidance on how to do this. None of the CPGs addressed preferences of family members/ companions (Fig. 4).

\section{Weighing benefits/harms and therapy prioritization}

Twenty-eight percent (13/47) of the CPGs mentioned weighing up the benefits and harms of CVD prevention for older people (see Table 2) and only one CPG explicitly mentioned the importance of prioritizing treatment in older people, albeit as a side issue.

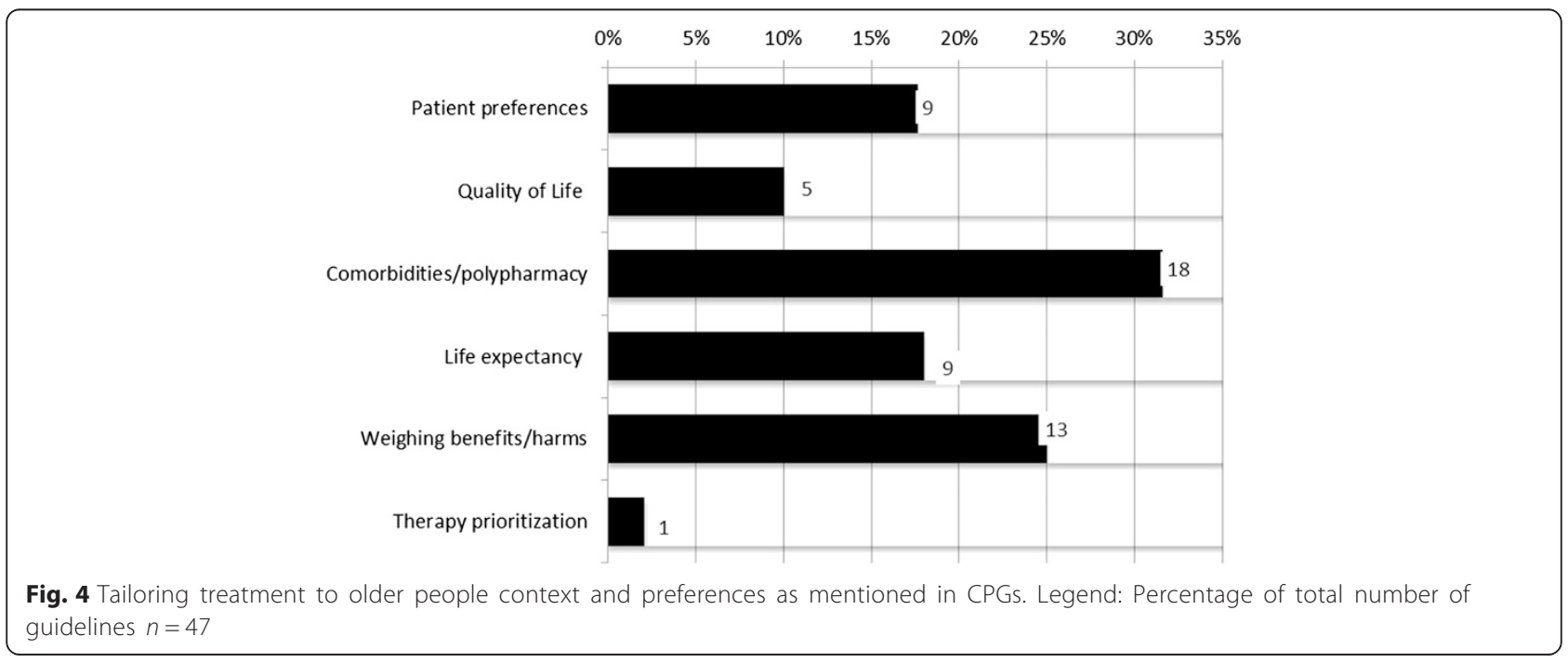




\section{Deprescribing}

Discontinuation of medication in older people was not considered in any of the CPGs, although one CPG explicitly mentioned that "(...) there is no reason for interrupting a successful and well tolerated therapy when a patient reaches 80 years of age [40]."

\section{Discussion}

Ninety-two percent of the included CPGs (43/47) referred to older adults to some extent, but the specific issues important in deciding about primary cardiovascular disease (CVD) prevention in older patients were mostly not adequately addressed. There was very limited discussion of frail older people and older people with comorbidities, a group for whom management is particularly challenging due to potential drug-drug and disease-drug interactions and competing health priorities [10,43]. Only $55 \%$ of the CPGs discussed available evidence for primary CVD prevention in older people and knowledge gaps. Potential benefits (in terms of morbidity, mortality and improved cognition) were discussed more extensively than harms (e.g. risk of hypotension with blood pressure medication), especially for hypertension medication and lifestyle recommendations. This is an important finding as even though older peoples' preferences to take medication for primary CVD prevention vary widely [15] they are relatively insensitive to its benefit but highly sensitive to its adverse effects [14, 44], suggesting that clinical CPGs need to place emphasis on both benefits and harms, especially for older people. More specific adverse effects were mentioned for cholesterol medication than blood pressure medication, for example only a few CPGs mentioned an increased risk of fall injuries as a result of blood pressure medication in older people [31]. Evidence for lifestyle management in older adults was brief in most CPGs, with generally no information provided on the specific benefits or effects on CVD or other outcomes for older people, the amount of lifestyle change needed to benefit, or differences between age groups. None of the guidelines made recommendations about when treatment could or should be stopped. Use of medication to prevent CVD in older people should be carefully monitored as the benefit-harm trade-off may change if people, for example, become increasingly frail [45].

Although the majority of the included CPGs provided one or more statements describing under which circumstances the CPG might be difficult to implement for older people, addressing issues such as lack of validation of the risk models, increased risk side of effects, and the need to adjust treatment targets, recommendations are varied and brief. Given the heterogeneity of the older population, and widely varying treatment (outcome) preferences, treatment decision making needs to be tailored to the specific circumstances of the individual older adult, taking into account their preferences and values [4, 20]. Half of the CPGs recommended clinicians to apply the CPGs with consideration of individual older patient context (e.g. comorbidities, life expectancy), but did not offer specific guidance. Even fewer CPGs mentioned the importance of older patient involvement in decision making and none of the CPGs mentioned the importance of family members/ companions.

\section{How our findings compare with other CPG assessments}

Consistent with our findings, reviews of Canadian [37] and Australian [36] CPGs on prevalent chronic conditions in the older population found that although most CPGs refer to the older population to some extent, only a handful of them adequately address issues related to older patients, especially patients with comorbidities. One recent review of CPGs for patients with type-2 diabetes mellitus showed that the impact of multiple comorbidities, patient's socio personal contexts, and patients' personal values and preferences were only narrowly addressed in most CPGs [46]. Although this review did not focus on older people specifically, it concurs with the limited attention to older patient context and patient involvement the CPGs included in the current study, and suggests it is an important issue with CPGs more broadly.

\section{Limitations and strengths}

The main limitation of our work is that we only looked at statements specifically referring to older people and it therefore does not allow for a comparison between recommendations for older versus younger adults. It also means that we did not include statements related to comorbidities or frailty that may have been applicable to older people, if they did not specifically address this group. However, we know from previous studies that CPGs rarely consider patients with comorbidities $[36,37,46]$ so it is unlikely that including more general statements would have changed our results. Moreover, from the viewpoint of a clinician having to decide about the management of an older patient, guidelines would need to be clearly signposted as relevant for older people rather than requiring extraction from general text about management. Since our main objective was to examine the extent to which CVD CPGs address older adults, we did not carry out a formal quality evaluation of the CPGs. We limited our eligibility criteria to CPGs published in English.

Finally, we did not include the recently published JBS3 [47], NICE CG181 [48] and NHLBI JNC8 CPG [49] in the review as these were published after our last search date (31 December 2013). However, these CPGs appear to have a similar pattern to our main findings, with the exception of NICE CG181. In both the JBS3 and JNC8 guidelines, the discussion of older people is mainly limited to a recommendation to adjust treatment targets and/or 
thresholds The JBS8 has added an adjusted treatment threshold for people aged $\geq 80$ years $(\mathrm{BP}<150 / 90 \mathrm{~mm} \mathrm{Hg}$, or $<150 / 85 \mathrm{~mm} \mathrm{Hg}$ if ambulatory or home BP monitoring is used). JNC8 now recommends an adjusted treatment threshold and target for people aged $\geq 60$ years (SBP of $150 \mathrm{mmHg}$ or DBP of $90 \mathrm{mmHg}$ ). Interestingly, the section on hypertension in older people in JNC7 guideline was removed in the JNC8 update. The updated NICE CG181 guideline provides much more extensive discussion of CVD risk management in older people than the previous version (CG67) [39]. In CG67 the main recommendation for older people had been to use clinical judgement to assess risk in people aged 75 or older but that this group could be considered at increased risk of CVD and is likely to benefit from statin treatment. In CG181 the major change is to explicitly recommend using the QRISK2 risk assessment, which has been validated in people up to, and including age 84 years. People aged 85 or older are considered to be at increased risk of CVD because of age alone and the CPG recommends considering statin treatment in this group. However, detail is added to make it explicit that there is limited evidence in older age groups, that the benefit may only be in reduced non-fatal MI and that consideration of risk and benefits and factors such as polypharmacy, comorbidity, frailty and life expectancy and informed patient preference are particularly important. It is also pointed out that there is a need for more research on the effectiveness of statin therapy in older people. The main strengths of our work includes the systematic approach, the use of a literature based data extraction method and the rigor of our analysis by employing double coding of CPG selection and data extraction.

\section{Implications for CPG development and research}

Clinical practice guidelines set out a template for best practice for patient management to guide and support clinicians in their care of patients. It is clear from our review that even though clinicians report wanting additional advice about caring for older patients, the current CPGs fail to provide adequate guidance in the vast majority of cases. There is a clear need for randomized trials on primary CVD prevention in older people (with inclusion of the frail older person with comorbidities) measuring outcomes that are meaningful to older people, to enable evidence based decision making for this group. At the same time, our results show that the majority of the CPGs do not even report the evidence that is available [27, 28, 31-34], rendering it almost impossible for older patients and their clinicians to make an informed decision about whether and how to manage CVD risk. CPG developers should think about ways to present available evidence for older people in the CPGs or at least to highlight the uncertainty around the evidence for this group. CPGs should be structured so that they may be easily adapted to older people with comorbidities, clearly present potential benefits and harms to allow for comparison, and are conducive to shared decision making. This may require the use of new technology [50]. Existing guidance on risk communication [51] (although mainly limited to younger people), use of prognostic indices [12] and health outcome prioritization tools $[13,15]$ for decision making in older people could help inform this process.

\section{Conclusion}

This study shows that only few primary CVD prevention CPGs adequately address important issues common in the care of older people. Given the changing demographics and aging population worldwide, this is of major significance. Future CPGs should provide more detailed evidence and guidance on the management of older people with multiple comorbidities and frailty, to ensure that discussions about the risk and benefits (and uncertainties) of different management options can take place and clinician and patients can make a shared decision that is tailored to the individual patient's circumstances and preferences. CPGs must be designed to provide the best possible advice and support to clinicians for the care of their patients. Care of older patients will not improve until important aspects of their care are addressed by health care providers. Achieving high quality guidelines to support clinicians is an essential part of this process.

\section{Additional files}

Additional file 1: Appendix A. Overview CPG recommendations older people $(n=47)$, organised by medical condition then by year of publication (reverse chronological) (PDF $354 \mathrm{~kb}$ )

Additional file 2: Appendix B. Examples of brief and more extensive guideline recommendations for older people according to the three criteria for CPG recommendations older people. (PDF $525 \mathrm{~kb}$ )

Additional file 3: Appendix C. Supporting Quotes from the CPGs (PDF $436 \mathrm{~kb})$

\section{Abbreviations}

AR: Absolute risk; CPG: Clinical Practice Guideline; CVD: Cardiovascular Disease; DBP: Diastolic blood pressure; SBP: Systolic blood pressure.

\section{Competing interests}

The authors declare that they have no competing interests.

\section{Authors' contributions}

All the authors included in the paper fulfil the criteria of authorship. JJ contributed to study design, analysis, interpretation, drafting, and revising the manuscript. SM contributed to data collection, analysis, interpretation, and revising the manuscript. CB contributed to analysis, interpretation, and revising the manuscript. $L I, J D, P G, B V M$ and $K M$ contributed to study design, interpretation, and revising the manuscript. BN contributed to data collection, interpretation, and revising the manuscript. All authors approved the final version of the manuscript and all authors are guarantors.

\section{Acknowledgements}

The authors would like to thank Ruth Mitchell for her help with the literature search. The study was funded by a Program Grant awarded to the Screening and Test Evaluation Program (STEP; 633003) from the National Health and 
Medical Research Council (NHMRC). CB was supported by an Australian Postgraduate Award. JJ (1037028) and KM (1029241) were supported by NHMRC fellowships. Jenny Doust was supported by the NHMRC project grant 511217. The funders had no role in the design or conduct of the study; in the collection, analysis, and interpretation of the data; or in the preparation or approval of the manuscript.

\section{Author details}

'Screening \& Test Evaluation Program (STEP), Sydney School of Public Health, The University of Sydney, Edward Ford Building A27, Sydney NSW 2006, Australia. ${ }^{2}$ Centre for Medical Psychology and Evidence-based Decision-making (CeMPED), The University of Sydney, Edward Ford Building A27, Sydney NSW 2006, Australia. ${ }^{3}$ Faculty of Health Sciences and Medicine, Bond University, Robina QLD 4226, Australia. ${ }^{4}$ Academic Medical Centre, Department of Internal Medicine, University of Amsterdam, Meibergdreef 9, 1105 AZ Amsterdam, Netherlands. ${ }^{5}$ Department of Geriatrics, Gelre Hospitaal, Albert Schweitzerlaan 31, 7334 DZ Apeldoorn, Netherlands.

\section{Received: 10 February 2015 Accepted: 22 July 2015}

Published online: 20 August 2015

\section{References}

1. Boyd CM, Darer J, Boult C, Fried LP, Boult L, Wu AW. Clinical practice guidelines and quality of care for older patients with multiple comorbid diseases: implications for pay for performance. Jama. 2005;294:716-24.

2. Scott IA, Guyatt GH. Cautionary tales in the interpretation of clinical studies involving older persons. Arch Intern Med. 2010;170:587-95.

3. Nair BR. Evidence based medicine for older people: available, accessible, acceptable, adaptable? Australas J Ageing. 2002;21:58-60.

4. American Geriatrics Society. Expert Panel on the Care of Older Adults with Multimorbidity. Patient-centered care for older adults with multiple chronic conditions: a stepwise approach from the American Geriatrics Society. J Am Geriatr Soc. 2012;60:1957-68.

5. Islam MM, Valderas JM, Yen L, Dawda P, Jowsey T, McRae IS. Multimorbidity and comorbidity of chronic diseases among the senior Australians: prevalence and patterns. PLoS One. 2014;9, e83783.

6. Barnett K, Mercer SW, Norbury M, Watt G, Wyke S, Guthrie B. Epidemiology of multimorbidity and implications for health care, research, and medical education: a cross-sectional study. Lancet. 2012;380:37-43.

7. National Seniors Productive Ageing Centre. Senior Australians and prescription medicines: usage, sources of information and affordability. Canberra: Department of Health and Ageing; 2012.

8. National Center for Health Statistics. Health, United States: 2013: With Special Feature on Prescription Drugs. Hyattsville, MD: U.S. Department of Health and Human Services; 2014.

9. Gnjidic D, Hilmer SN, Blyth FM, Naganathan V, Waite L, Seibel MJ, et al. Polypharmacy cutoff and outcomes: five or more medicines were used to identify community-dwelling older men at risk of different adverse outcomes. J Clin Epidemiol. 2012;65:989-95.

10. Tinetti ME, Bogardus Jr ST, Agostini JV. Potential pitfalls of disease-specific guidelines for patients with multiple conditions. N Engl J Med. 2004;351:2870-4.

11. Holmes HM, Hayley DC, Alexander GC, Sachs GA. Reconsidering medication appropriateness for patients late in life. Arch Intern Med. 2006;166:605-9.

12. Yourman LC, Lee SJ, Schonberg MA, Widera EW, Smith AK. Prognostic indices for older adults: A systematic review. JAMA. 2012;307:182-92.

13. Fried TR, Tinetti ME, lannone L, O'Leary JR, Towle V, Van Ness PH. Health outcome prioritization as a tool for decision making among older persons with multiple chronic conditions. Arch Intern Med. 2011;171:1854-6.

14. Fried TR, McGraw S, Agostini JV, Tinetti ME. Views of older persons with multiple morbidities on competing outcomes and clinical decision-making. J Am Geriatr Soc. 2008;56:1839-44.

15. Tinetti ME, MCAvay GJ, Fried TR, Allore HG, Salmon JC, Foody JM, et al. Health outcome priorities among competing cardiovascular, fall injury, and medication-related symptom outcomes. J Am Geriatr Soc. 2008;56:1409-16.

16. Fried TR, O'Leary J, Van Ness P. Fraenkel L. Inconsistency over time in the preferences of older persons with advanced illness for life-sustaining treatment. J Am Geriatr Soc. 2007;55:1007-14.

17. Chewning B, Bylund CL, Shah B, Arora NK, Gueguen JA, Makoul G. Patient preferences for shared decisions: a systematic review. Patient Educ Couns. 2012;86:9-18.
18. Arora NK, McHorney CA. Patient preferences for medical decision making: who really wants to participate? Med Care. 2000;38:335-41.

19. Laidsaar-Powell RC, Butow PN, Bu S, Charles C, Gafni A, Lam WWT, et al. Physician-patient-companion communication and decision-making: A systematic review of triadic medical consultations. Patient Educ Couns. 2013;91:3-13.

20. van der Weijden T, Pieterse AH, Koelewijn-van Loon MS, Knaapen L, Légaré F, Boivin A, et al. How can clinical practice guidelines be adapted to facilitate shared decision making? A qualitative key-informant study. BMJ Quality \& Safety. 2013;22:855-63.

21. Petersen LK, Christensen K, Kragstrup J. Lipid-lowering treatment to the end? A review of observational studies and RCTs on cholesterol and mortality in $80+-$ year olds. Age Ageing. 2010;39:674-80.

22. Gu Q, Burt VL, Dillon CF, Yoon S. Trends in antihypertensive medication use and blood pressure control among United States adults with hypertension. The National Health and Nutrition Examination Survey, 2001 to 2010. Circulation. 2012;126:2105-14

23. Ferket BS, Colkesen EB, Visser JJ, Spronk S, Kraaijenhagen RA, Steyerberg EW, et al. Systematic review of guidelines on cardiovascular risk assessment: which recommendations should clinicians follow for a cardiovascular health check? Arch Intern Med. 2010;170:27-40.

24. D'Agostino RB, Vasan RS, Pencina MJ, Wolf PA, Cobain M, Massaro JM, et al. General cardiovascular risk profile for use in primary care the Framingham Heart Study. Circulation. 2008;117:743-53.

25. Jackson R. Cardiovascular risk prediction: are we there yet? Heart. 2008;94:1-3.

26. Liew SM, Jackson R, Mant D, Glasziou P. Should identical CVD risks in young and old patients be managed identically? Results from two models. BMJ Open. 2012;2, e000728.

27. Beckett NS, Peters R, Fletcher AE, Staessen JA, Liu L, Dumitrascu D, et al. Treatment of hypertension in patients 80 years of age or older. $\mathrm{N}$ Engl J Med. 2008;358:1887-98.

28. Beckett N, Peters R, Tuomilehto J, Swift C, Sever P, Potter J, et al. Immediate and late benefits of treating very elderly people with hypertension: results from active treatment extension to hypertension in the very elderly randomised controlled trial. BMJ. 2012;344:d7541.

29. Baigent C, Keech A. Kearney PMa, Blackwell L, Buck G, Pollicino C, Kirby A, Sourjina T, Peto R, Collins R, Cholesterol Treatment Trialists'(CTT) Collaborators. Efficacy and safety of cholesterol-lowering treatment: prospective meta-analysis of data from 90,056 participants in 14 randomised trials of statins. Lancet. 2005;366:1267-78.

30. Savarese G, Gotto Jr AM, Paolillo S, D'Amore C, Losco T, Musella F, et al. Benefits of statins in elderly subjects without established cardiovascular disease: a meta-analysis. J Am Coll Cardiol. 2013;62:2090-9.

31. Tinetti ME, Han L, MCAvay GJ, Lee DSH, Peduzzi P, Dodson JA, et al. Anti-hypertensive medications and cardiovascular events in older adults with multiple chronic conditions. PLoS One. 2014;9, e90733.

32. Hilmer SG, Gnjidic D. Statins in older adults. Aust Prescr. 2013;36:79-82

33. Naganathan V. Cardiovascular drugs in older people. Aust Prescr. 2013;36:190-4

34. Butt DA, Mamdani M, Austin PC, Tu K, Gomes T, Glazier RH. The risk of falls on initiation of antihypertensive drugs in the elderly. Osteoporos Int 2013;24:2649-57.

35. Weiner M, Wells S, Kerse N. Perspectives of general practitioners towards evaluation and treatment of cardiovascular disease among older people. J Primary Health Care. 2009;1:198-206.

36. Vitry Al, Zhang Y. Quality of Australian clinical guidelines and relevance to the care of older people with multiple comorbid conditions. Med J Aust. 2008;189:360-5.

37. Mutasingwa DR, Ge H, Upshur REG. How applicable are clinical practice guidelines to elderly patients with comorbidities? Can Fam Physician. 2011;57:e253-62.

38. Moschetti I, Brandt D, Perera R, Clarke M, Heneghan C. Adequacy of reporting monitoring regimens of risk factors for cardiovascular disease in clinical guidelines: systematic review. BMJ. 2011;342:d1289.

39. National Institute for Health and Clinical Excellence. NICE Clinical Guideline 67: Lipid modification - Cardiovascular risk assessment and the modification of blood lipids for the primary and secondary prevention of cardiovascular disease. London: National Institute for Health and Clinical Excellence; 2010.

40. The Task Force for the Management of Arterial Hypertension of the European Society of Hypertension and of the European Society of Cardiology. 2007 Guidelines for the management of arterial hypertension. Eur Heart J. 2007;28:1462-536. 
41. Rothwell PM, Price JF, Fowkes FGR, Zanchetti A, Roncaglioni MC, Tognoni G, et al. Short-term effects of daily aspirin on cancer incidence, mortality, and non-vascular death: analysis of the time course of risks and benefits in 51 randomised controlled trials. Lancet. 2012;379:1602-12.

42. National Institute for Health and Clinical Excellence. NICE Clinical Guideline 127: Hypertension. Clinical management of primary hypertension in adults. London: National Institute for Health and Clinical Excellence; 2011.

43. McMillan GJ, Hubbard RE. Frailty in older inpatients: what physicians need to know. QJM. 2012;105:1059-65.

44. Fried TR, Tinetti ME, Towle V, O'Leary JR, lannone L. Effects of benefits and harms on older persons' willingness to take medication for primary cardiovascular prevention. Arch Intern Med. 2011;171:923-8.

45. Garfinkel D, Mangin D. Feasibility study of a systematic approach for discontinuation of multiple medications in older adults: addressing polypharmacy. Arch Intern Med. 2010;170:1648-54.

46. Wyatt KD, Stuart LM, Brito JP, Carranza Leon B, Domecq JP, Prutsky GJ, et al. Out of context: clinical practice guidelines and patients with multiple chronic conditions: a systematic review. Med Care. 2014;52 Suppl 3:S92-s100.

47. JBS3 Board. Joint British Societies' consensus recommendations for the prevention of cardiovascular disease (JBS3). Heart. 2014;100:ii1-ii67.

48. National Institute for Health and Care Excellence. NICE Clinical Guideline CG181:Lipid modification: Cardiovascular risk assessment and the modification of blood lipids for the primary and secondary prevention of cardiovascular disease. London: National Institute for Health and Care Excellence; 2014

49. James PA, Oparil S, Carter B, Cushman W, Dennison-Himmelfarb C, Handler J, et al. 2014 Evidence-based guideline for the management of high blood pressure in Adults. Report from the panel members appointed to the Eighth Joint National Committee (JNC 8). JAMA. 2014;311:507-20.

50. Guthrie B, Payne K, Alderson P, McMurdo MET, Mercer SW. Adapting clinical guidelines to take account of multimorbidity. BMJ. 2012;345, e6341.

51. Trevena LJ, Zikmund-Fisher BJ, Edwards A, Gaissmaier W, Galesic M, Han PKJ et al. Presenting quantitative information about decision outcomes: a risk communication primer for patient decision aid developers. BMC Med Inform Decis Mak. 2013;13:S7

\section{Submit your next manuscript to BioMed Central and take full advantage of:}

- Convenient online submission

- Thorough peer review

- No space constraints or color figure charges

- Immediate publication on acceptance

- Inclusion in PubMed, CAS, Scopus and Google Scholar

- Research which is freely available for redistribution

Submit your manuscript at www.biomedcentral.com/submit 